\title{
Protective ocular mechanisms in woodpeckers
}

\begin{abstract}
Aims Woodpeckers possess mechanisms protecting the eye from shaking/impact. Mechanisms available to woodpeckers but not humans may help explain some eye injuries in Shaken Baby syndrome (SBS).

Methods Gross dissection and histologic examination of eyes and orbits of seven woodpeckers.

Results All birds showed restricted axial globe movement due to the tight fit within the orbit and fascial connections between the orbital rim and sclera. The sclera was reinforced with cartilage and bone, the optic nerve lacked redundancy, and the vitreous lacked attachments to the posterior pole retina. Conclusions Woodpecker eyes differ from human infants by an inability of the globe to move axially in the orbit, the sclera to deform, and the vitreous to shear the retina. These findings support current hypotheses that abusive acceleration-deceleration-induced ocular injury in human infants may be related to translation of vitreous within the globe and the globe within the orbit. The woodpecker presents a natural model resistant to mechanical forces that have some similarity to SBS.

Eye (2007) 21, 83-89. doi:10.1038/sj.eye.6702163; published online 18 November 2005
\end{abstract}

Keywords: woodpeckers; shaken baby syndrome; protective mechanisms

\section{Introduction}

Retinal haemorrhages are one of the principle manifestations of Shaken Baby syndrome (SBS), occurring in $50-100 \%$ of cases. ${ }^{1-5}$ Other ocular findings include schisis of the macula induced by vitreous traction, optic atrophy, and less commonly, retinal detachment. Retinal haemorrhages in SBS are thought to be in part the result of acceleration-deceleration-induced
T Wygnanski-Jaffe ${ }^{1}$, CJ Murphy², C Smith ${ }^{3}$, M Kubai $^{2}$, P Christopherson ${ }^{4}$, CR Ethier ${ }^{5}$ and AV Levin ${ }^{1}$

shearing forces as the vitreous moves within the globe and the globe moves within the orbit. ${ }^{6}$ Impact amplifies the acceleration-decelerationinduced forces.?

Animal models for SBS have been created by laboratory shaking of rats, mice, pigs, and cats. Most researchers examined the brains without looking at the eyes or orbits. ${ }^{8,9}$ In murine models, when eye examination was conducted, retinal haemorrhage may or may not be seen. ${ }^{10}$ The woodpecker presents a naturally occurring model resistant to mechanical forces similar to those encountered in SBS, and we therefore reasoned that insight into the pathophysiology of SBS could be gained from understanding mechanisms in the woodpecker eye that protect it from injury during rapid accelerationdeceleration while pecking.

\section{Materials and methods}

12 prepared adult woodpecker skulls representing five species collected from a variety of sources were examined to assess the orientation and structure of the orbit within the skull. All skulls were photographed from at least three orientations with a metric ruler (Figure 1).

Seven dead woodpeckers were collected from parts of Wisconsin by the School of Veterinary Medicine, University of Wisconsin, Madison campus. All seven birds had been collected after death from a variety of causes: two tower strikes, one hawk kill, four unknown. The woodpeckers represented three species: two flickers (Colaptes auratus), two red-headed woodpeckers (Melanerpes erythrocephalus), and three yellow bellied sapsuckers (Sphyrapicus varius).

All birds were immersion fixed in $10 \%$ neutral buffered formalin for periods ranging from 3 days to 6 months. Using loupes, we performed macroscopic dissection of the fascial attachments between the anterior portions of the globe and surrounding structures using a
${ }^{1}$ Department of Ophthalmology and Vision Sciences, M158, The Hospital for Sick Children, 555 University Avenue, Toronto, Ontario, Canada M5G 1X8

${ }^{2}$ Department of Surgical Sciences, School of Veterinary Medicine, University of Wisconsin, Madison, WI, USA

${ }^{3}$ Department of Pathology and Laboratory Medicine, The Hospital for Sick Children, University of Toronto, Toronto, Canada

${ }^{4}$ Department of Pathobiology, College of Veterinary, Medicine, Auburn University, Auburn, AL, USA

${ }^{5}$ Departments of Mechanical and Industrial Engineering and Ophthalmology, University of Toronto, Toronto, Canada

Correspondence: AV Levin, Tel: + 14168131076 ; Fax: + 14168136261

E-mail: alex.levin@ sickkids.ca

Received: 31 May 2005 Accepted in revised form: 17 September 2005 Published online: 18 November 2005 


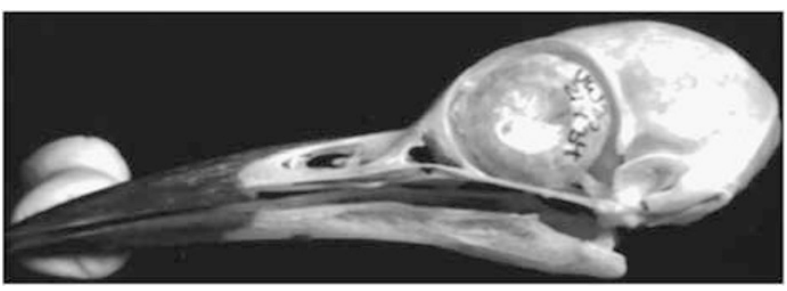

Figure 1 Woodpecker skull intraocular bony ossicle (specimen is labelled with writing on the ossicle) is anterior to orbital rim. Orbit is oriented such that visual axis is approximately $75^{\circ}$ from saggital plane.

scalpel blade, scissors, and ronjeurs. In one bird, the globe was approached by first invading the intracranial space and dissecting forward so that the orbit was opened from behind and above. After we inspected the relationship of the globe and optic nerve to the bony orbit, the eyes were enucleated. The extraocular muscles were identified and used for orientation of the globe. Five of the dissected eyes were then decalcified for 30-60 min using Decal I (Surgipath Medical Industries Inc., Richmond, IL, USA) and inspected under a dissecting microscope after dividing the globe into two calottes at $150^{\circ}$, below the optic nerve. These five eyes were then routinely processed for paraffin embedding and subsequently sectioned at $8 \mu \mathrm{m}$ for histologic examination. Sections were stained with haematoxylin and eosin, Masson trichrome or alcian blue, and periodic acid Schiff. All steps of the gross and microscopic dissections were photographed. Five dissections were performed at the University of Wisconsin School of Veterinary Medicine by the authors (TWJ, CJM, AVL) with two additional birds being dissected at The Hospital for Sick Children by one or two authors (TWJ, AVL). Slight variations on the above technique were used for the latter birds, since they were used for the purpose of identifying the angle of the visual axes, optic nerve lengths, and axial globe lengths.

\section{Results}

We found no significant ocular or orbital variations among the seven different species of woodpeckers. Examination of gross specimens as well as the prepared skulls revealed that, the woodpecker orbits take up most of the skull and have a far greater volume than the brain cavity, which is posterior and medial to the orbits. The two globes are separated at the midline by a thin (approx. $0.1 \mathrm{~mm}$ ) bony interorbital septum. The globe is supported inferio-laterally by the bony quadrate process (Figure 1). The quadrate bone suspends the lower jaw in nonmammalian vertebrates and represents the evolutionary forerunner of an inner ear bone (the incus) in mammals. The orbital floor consists of a firm membrane that runs

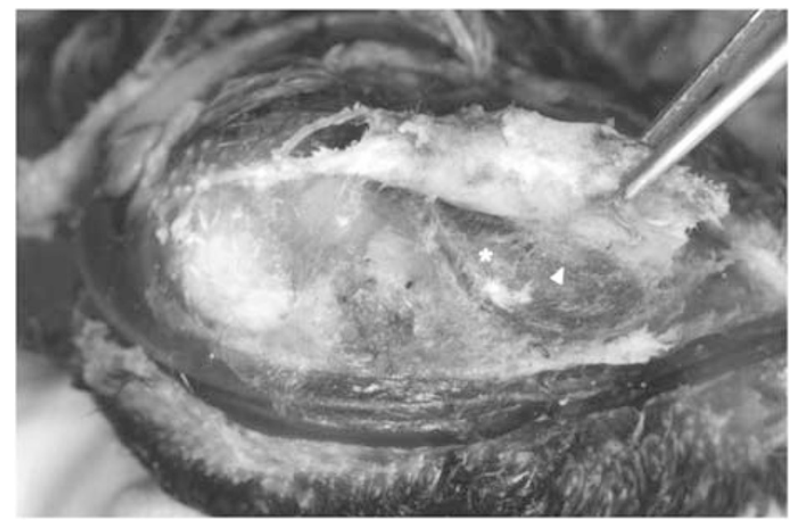

Figure 2 Tight fascial connections between the eye, orbit and surrounding tissues, encasing the woodpecker eye and anchoring the globe to surrounding structures. Asterix indicates level of the scleral ossicle. Arrow indicates cornea.

medially from the quadrate process. The globe is oriented in the skull such that the visual axis makes an angle of approximately $75^{\circ}$ relative to the sagittal plane, placing the posterior pole of the retina tangential to the anterior-posterior plane of the strike.

In fixed specimens the palpebral fissure is narrow and allows exposure of less than $50 \%$ of the corneal surface. It is exposed to a greater degree in life. On dissection of the periocular tissues we found that a shallow cone of extensive very adhesive fascial interconnections involved the periorbita, the orbital rim and the sclera. These were the subocular ligament, the subocular

laryngomandibular ligament, and the postorbital ligaments. Although the prolonged formalin fixation made fine gross dissection of the tissues difficult, we found that virtually the majority of the bulbar surface of the lids, beginning at the conjunctival fornices and extending peripherally, is intimately related and attached to the underlying sclera from the orbital rim forward (Figure 2). Only the regions extending 1-2 mm peripherally from the upper and lower lid margins are free of these fascial attachments. This causes the anterior portion of the globe to be bound firmly to the overlying lids and orbital rim.

Laterally, the medial aspect of the globe at the plane of the ora serrata is actually well within the orbital limits. The orbital rim lies just posterior to the posterior margin of the scleral ossicles (Figure 1). The scleral ossicles are small bones in the anterior sclera that unite to form a ring that extend from just posterior to the limbus to the equatorial region of the globe. The scleral ossicles are a constant anatomic feature in the eyes of all birds. They determine the shape of the eye, serve as firm anchors for the origin of the striated ciliary muscles and provide protection to the lateral aspect of the globe that lies largely outside of any bony orbital protection. The 
nictitating membrane covers the eye inferiorly and medially during rest and passes laterally across the globe.

Macroscopic dissection of the orbit after the lids, periorbita, and superior orbital rims were removed, revealed that the globe enlarges dramatically behind the palpebral apertures such that the posterior segment of the eye has a cross-sectional width that is remarkably larger than the anterior segment. The globe is situated tightly within the orbit and the posterior segment is closely apposed to the bony orbital walls. Therefore, it appears that translational movement of the globe in the orbit would be severely limited. The optic nerve is straight with no redundancy. In the birds that were measured, the mean axial length of the eye was $7.5 \mathrm{~mm}$ and the intraorbital length of the optic nerve was $<1 \mathrm{~mm}$.

Only after enucleation could we demonstrate the six extraocular muscles directly adherent to the posterior sclera (Figure 3). The woodpecker has four rectus muscles, and an inferior and superior oblique muscle, which move the globe. In addition, the pyramidalis and quadratus muscles originate from the posterior surface of the globe and act in concert to effect movement of the nictitating membrane. The tendon of the pyramidalis muscle passes through the aponeurotic sleeve-like insertion of the quadratus muscle. The tendon encircles the optic nerve and passes inferiorly and then anterior in route to its insertion on the inferomedial aspect of the nictitan. When the quadratus muscle contracts it acts to amplify the action of the pyramidalis muscle and thereby effects movement of the nictitans. Unlike humans, they possess no muscular cone running parallel to the optic nerve. Rather, the extraocular muscle orientations from orbital wall to insertion on the sclera (excepting the quadratus and pyramidalis muscles) have a 'plate-like'

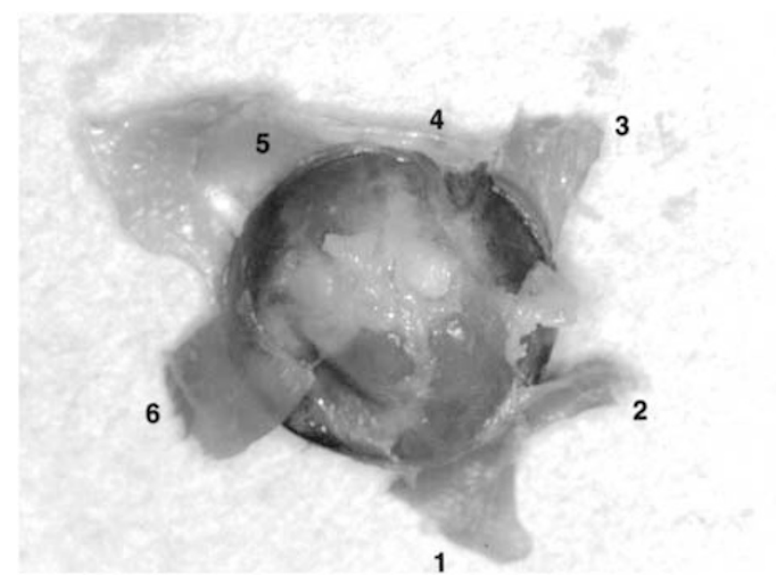

Figure 3 Extraocular muscles of an enucleated woodpecker eye. (1) Superior rectus muscle (2) superior oblique muscle (3) medial rectus muscle (4) inferior oblique muscle (5) inferior rectus muscle (6) lateral rectus muscle. configuration lying completely tangential to the sclera. These muscles effect rotational movements of the eye, although the degree of ocular movement would be limited by the firm fascial attachments of the sclera to the periorbita.

After enucleation, decalcification and sectioning of the globes, we were able to identify the pecten; a pigmented vascular structure which emanates from the optic nerve head extending forward into the vitreous. Woodpeckers, similar to other avian species do not have retinal vessels. The pecten is thought to provide nutrition to the internal layers of the retina. Although the vitreous was degenerated in all specimens examined, the woodpecker vitreous appears to have a more solid consistency than the viscous liquid of the formalin-fixed human vitreous based on a qualitative observation of primarily fixed specimens. Although, ideally measurements of fresh vitreal samples from both adult and infant humans as well as from woodpeckers to address this issue would provide firmer confirmation, these were not available. We were able to observe that the vitreous is attached to the apex of the pecten (Figure 4). No vitreous

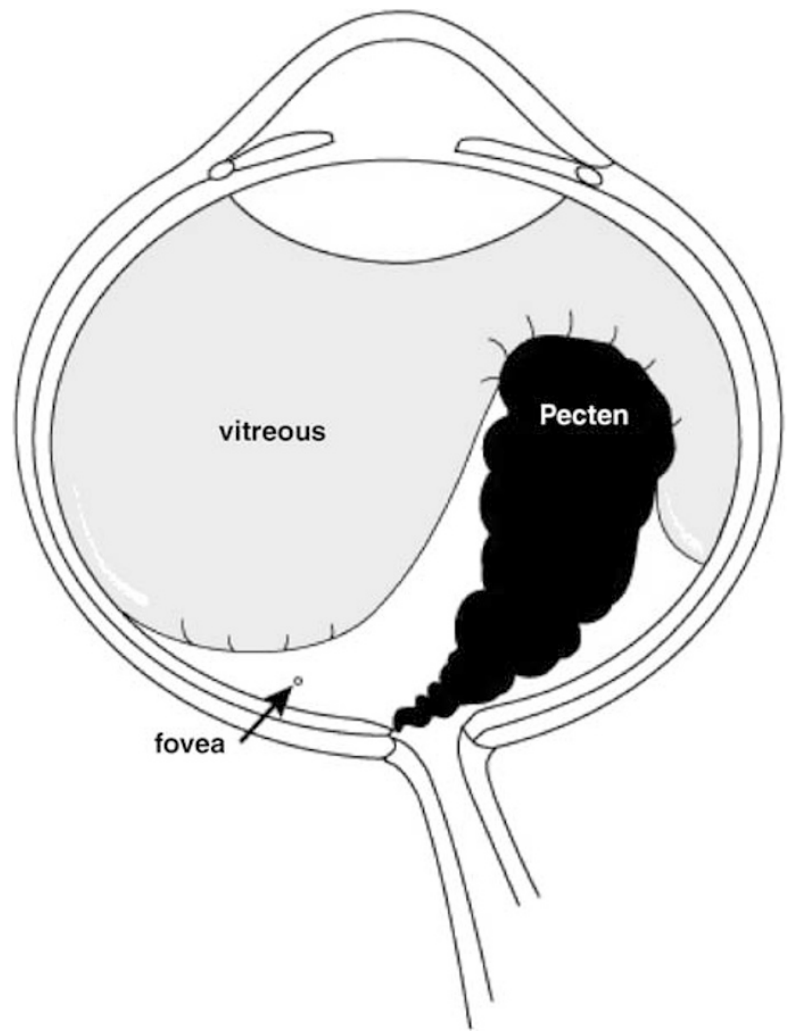

Figure 4 Schematic illustration of the woodpecker eye demonstrating the vascular pecten which is attached only to the optic nerve area. The vitreous drapes over the pecten with attachments to the pecten apex but not to the posterior pole. This illustration does not display the presence of the anterior scleral ossicles or posterior scleral cartilage characteristic of birds. 
attachments were observed between the vitreous and posterior pole although it appeared that the vitreous was apposed to the peripheral retina for $360^{\circ}$. In all specimens the cornea was collapsed (Figure 2) and in four cases, the lens was found to lie on the face of the collapsed vitreous body in the posterior segment. We believe this is due to poor fixation as well as the decalcification process. No bird had evidence of direct blunt trauma to the globe.

Under histologic examination, we observed that the scleral ring consists of compact bone. The ossicular ring, composed of numerous interdigitating bones is located intrasclerally at the level of the ciliary body. In addition, intrascleral cartilage is present posteriorly. The region of overlap between the scleral ossicles and the posteriorly situated cartilage lies external to the region of the ora serrata. This intrinsic scleral cartilage lamina lies within the sclera throughout its extent posterior to the ora.

\section{Discussion}

Woodpeckers peck with remarkable intensity and speed. ${ }^{11}$ Although no experimental studies have been carrried out to our knowledge, we presume that these birds are protected against acceleration-decelerationimpact-related eye trauma; otherwise there would be a plethora of poorly sighted or blind woodpeckers in nature, which is not the case. An increased prevalence of ocular lesions in woodpeckers has not been noted in the veterinary literature or in the literature relating to wildlife rehabilitation.

Our findings show that woodpeckers possess protective orbital and ocular mechanisms that may explain the lack of known shaking-impact-related eye trauma in these birds.

\section{Orbital structures}

We found that the woodpecker globe is 'tightly packed' in the orbit, which minimizes relative motion between the orbit and globe. This is accomplished by the presence of extensive fascial connections between the orbital rim and the globe, a relatively narrow orbital entrance compared to the size of the posterior segment, and a paucity of space between the globe and orbital walls. In addition, the attachments of the periorbita are to a part of the globe that is intrinsically rigid due to the scleral ossicles (there is one intrascleral ossicular ring in each eye comprised of interdigitating scleral ossicles) and intrascleral cartilage, further stabilizing the eye. Movement of the globe is effected by contraction of the extraocular muscles, which are tangential and adherent to the sclera without the presence of a true muscular cone. The anatomy of ocular and orbital structures predicts the globe to allow only limited rotational movement and resistance to translational movement along its axis. This is consistent with the structure of the optic nerve, which has a very short and straight intraorbital course, without the lax, S-shaped configuration seen in humans. It should be noted that this feature of the optic nerve is characteristic of birds in general (CJM unpublished observations).

In addition, videography shows that woodpeckers close their eyes immediately prior to each strike. ${ }^{12}$ This not only serves to prevent chips from entering the eye, but may also create a 'seat belt' restraining the globe even further during the intense impact deceleration. ${ }^{13}$

This situation is quite different than that seen in infants. The human orbit contains a sizable quantity of orbital soft tissue allowing motion of the globe when a human infant is subjected to abusive accelerationdeceleration forces (ie, SBS). The human intraorbital optic nerve is longer than the distance between the back of the globe and the orbital apex with an S-shaped configuration accounting for the excess. Since the orbital structures are firmly adherent to their entry sites into the sclera and at the orbital apex, violent translational movement of the globe could generate potentially injurious traction at these points of fixation.

Haemorrhage and injury to these structures, such as intrascleral haemorrhage at the optic nerve-sclera juncture, intradural haemorrhage in the optic nerve sheath posteriorly, or apical haemorrhage into orbital fat have been demonstrated in autopsies of victims of SBS victims. ${ }^{6,14-17}$ Stretching and traction injury to the optic nerve might also explain the appearance of optic nerve atrophy, one of the major causes of visual loss in the long-term follow-up of SBS victims. ${ }^{6}$ Accelerationdeceleration injury to the orbital contents in SBS may play a role in the generation of retinal haemorrhage through injury to blood vessels or autonomic neural structures destined for the globe. The latter are involved in retinal vascular autoregulation. Like retinal haemorrhages, ${ }^{6}$ these orbital findings are not commonly seen in fatal single impact accidental head trauma (AVL, data not shown).

\section{Intraocular structures}

In woodpeckers, the strong compact bone of the anteriorly situated scleral ossicles, along with the posterior intrascleral cartilage, converts the sclera from a pliable soft tissue to a less deformable coat. Human infant sclera is noteworthy for its decreased rigidity as compared to adults. ${ }^{18}$ From the fifth month of gestation to the age of 3 years the shape of the human sclera may be modified due to stretching of the elastic fibres in the sclera beyond their limit of elasticity when not supported 
by a fully developed collagenous structure. ${ }^{19}$ A deformable globe may allow more relative motion of intraocular components, causing humans to be more susceptible to injury from strong accelerationdeceleration-induced forces.

A second important difference between the woodpecker and human relates to vitreo-retinal coupling. In birds, the internal aspect of the avascular retina receives its nutrients and oxygen by diffusion from vessels lying within the pecten (the outer retina is supplied by the choroid), which is supplied by vessels running within the optic nerve. It is noteworthy that there is an absence of adhesions between the vitreous and the retina in the posterior pole of the woodpecker eye although the vitreous is attached to the apical bridge of the pecten. This 'draping' of the vitreous, has previously been observed in raptors. ${ }^{20}$ The choroid in woodpeckers may serve as a cushion with an unknown mucopolysaccharide filling its interstices. The pectin may also have a role in maintaining an effective cushion as the pectin can fill with blood to briefly elevate intraocular pressure thus possibly maintaining firm pressure on the lens and retina to prevent damage. ${ }^{21}$ Optical coherence tomography performed by one of the authors (CJM, data not shown) and co-workers has confirmed the absence of vitreous attachment to the posterior pole in the living red-tailed hawk (Buteo jamaicensis).

This relative lack of vitreo-retinal attachment is in contrast to the situation in children where the vitreous is tightly adherent to the retina at the posterior pole, blood vessels, and peripheral retina. Similarly, traction from a shaking vitreous may also be responsible for the predilection for peripheral ${ }^{6}$ and other types of retinal haemorrhage in SBS. In infants the vitreous is more adherent to the retina and more formed than in adults in which the vitreous undergoes a process of liquefaction with aging. These differences may also leave children more susceptible to retinal haemorrhages and traumatic retinoschisis due to acceleration-deceleration. ${ }^{6,22-25}$ Damage to intraocular components will occur when there is relative motion between intraocular components, leading to shearing, tearing, compression, and extension. Such relative motion will be generated during acceleration and deceleration when adjacent tissues are of different densities, and are tightly coupled to one another so as to allow relative motion. Shearing (tangential) motions are expected to be particularly dangerous at the vitreo-retinal interface, due to the layered structure of the retina. Further, shearing motions will be most pronounced when the plane of ocular structures coincides with the axis of accelerationdeceleration. In the woodpecker eye, the lack of coupling of the vitreous to the posterior pole, as well as the orientation of the eye with respect to the axis of striking, drastically reduces such relative shearing motions. This would reduce the possibility of shaking-induced retinal injury, such as traumatic retinoschisis.

\section{Comparison with other avian species}

Many of the ocular and orbital adaptations described for the woodpecker are present in all avian species. Specifically, the presence of intrascleral bone and cartilage is a hallmark of avian ocular anatomy. The lack of vitreal attachments to the retina at the posterior pole has only been explored in a few species. Although we did not conduct any systematic studies, nor were we able to locate any published accounts for comparison, it is worth noting that the periorbital fascia anchoring the globe to the orbit in all of the woodpeckers examined was more extensive than that found in members of the falconiformes (diurnal birds of prey) that do not display any appreciable pecking behaviour (CJM, unpublished observations). A unique feature of the woodpecker musculature is the presence of dense fibrous condensations within the hyoid musculature which, along with the musculature itself, has been suggested to act as a stabilizer during impact thereby diminishing intracranial trauma. ${ }^{26-30}$ It should be noted that the degree of development of these fibrous condensations is variable between species of woodpeckers. We did not systematically study this anatomic feature in our specimens.

All birds exhibit various degrees of pecking behaviour. From our studies, it seems that woodpeckers, rather than evolving a unique set of morphologic adaptations, have capitalized on these general avian features that, in combination with the unique hyoid musculature, allow execution of its uniquely powerful pecking behaviour. The absence of significant interspecies variation in the woodpeckers we studied supports this concept as pecking behaviours between species of woodpeckers can also vary considerably. For these reasons we believe that enlarging our sample size is unlikely to uncover new findings.

\section{Future work}

Future studies might involve live birds, attempting to surgically impair the presumed protective mechanisms (eg disruption of globe fixation by inducing orbital rim fracture) and then allowing the woodpecker to resume its striking behaviour with subsequent observation regarding induced ocular damage due to the unprotected state. However, woodpeckers are difficult to maintain in captivity due to their feeding behaviours. ${ }^{12}$ In addition, nonreleasable injured woodpeckers may exhibit reduced pecking behaviour as they develop other feeding adaptations in captivity (AVL, data not shown). 
Repeating our work with freshly euthanized woodpeckers would also be helpful as we found that prolonged formalin fixation creates challenges for fine tissue dissection particularly in the periorbita. Although we have obtained Federal and State licenses to collect live birds, this too has proven quite challenging.

Another problem with the woodpecker model is the absence of retinal vessels. Only mammals (with a few rare exceptions) have true intraretinal vessels. Many species have what appear to be retinal vessels that are actually epiretinal vessels as in frogs. We are currently planning to use a mechanical shaker with rats, focusing on orbital and ocular injury. It is also our intent to explore other possible natural models such as ground hogs that are shaken to death by dogs.

Acceleration-deceleration with or without blunt impact is a key element in the ocular insult of SBS. ${ }^{14-17}$ Shaken infants experience head movements in all planes due to their weak cervical musculature, proportionately larger head size, and perhaps an increasingly altered state of consciousness as brain injury ensues. Angular and rotational forces are particularly important in the generation of the characteristic injuries. ${ }^{31}$ Woodpeckers experience a pure anterior-posterior strike which is anticipated, so that its effects can be minimized by keeping the woodpecker's head in the plane of its body. In this way, the head does not rotate during pecking, in contrast to the situation in human infants undergoing violent shaking.

In addition the woodpecker may be protected to some extent by its small brain size resulting in a small ratio of brain weight to brain surface area. Any impact force would be spread out over a relatively large area making its brain somewhat more resistant to concussion than a human's brain. The infants head in contrast is proportionately much larger relative to the body and pivots on the neck accentuating the anterior-posterior movement causing injury. ${ }^{21}$

Although infant and woodpecker accelerationdeceleration are different in many aspects, our data offer possible indirect insights into the pathophysiology of eye injury in SBS. Our findings suggest that woodpeckers have protective adaptations that, by the absence of such mechanisms in human infants, support current theories regarding the potential injurious effect of repetitive acceleration-deceleration in generating the retinal haemorrhages and other forms of ocular injury seen in SBS through translation of the globe and orbital tissue injury, vitreo-retinal traction, and scleral deformation.

\section{Acknowledgements}

This study was completed with the valuable contributions of research assistants Enza Perruzza and
Erica Bell, and ophthalmic imaging specialist Leslie MacKeen. We also gratefully acknowledge Dr Randell Alexander who was the first to our knowledge, to suggest that woodpeckers could serve as a model for protective mechanisms for shaking impact injury.

\section{References}

1 Ludwig S, Warman M. Shaken baby syndrome: a review of 20 cases. Ann Emerg Med 1984; 13: 51-54.

2 Duhaime AC, Gennarelli TA, Thibault LE, Bruce DA, Margulies SS, Wiser R. The shaken baby syndrome: a clinical, pathological, and biomechanical study. J Neurosurg 1987; 6: 409-415.

3 Hadley M, Sonntag UK, Rekate HL, Murphy A. The infant whiplash-shake injury syndrome: a clinical and pathological study. Neurosurgery 1989; 24: 536-540.

4 Harcourt B, Hopkins D. Ophthalmic manifestations of the battered baby syndrome. Br Med J 1971; 3: 398-401.

5 Mushin A. Ocular damage in the battered-baby syndrome. Br Med J 1971; 3: 402-404.

6 Levin AV. Retinal hemorrhages and child abuse. In: David TJ (ed). Recent Advances in Paediatrics. Churchill Livingstone: London, 2000 pp 151-160.

7 Duhaime AC, Christian CW, Rorke LB, Zimmerman RA. Nonaccidental head injury in infants-the 'shaken baby syndrome'. N Engl J Med 1998; 338: 1822-1829.

8 Barron KD, Dentinger MP, Kimelber HK, Nelson LR, Bourke $\mathrm{RS}, \mathrm{Keegan} \mathrm{S}$ et al. Ultrastructural features of a brain injury model in the cat: I. Vascular and neuroglial changes and the prevention of astroglial swelling by a fluorenyl (aryloxy) alkanoic acid derivative (L-644,711). Acta Neuropathol (Berlin) 1988; 75: 295-307.

9 Smith SL, Andrus PK, Gleason DD, Hall ED. Infant rat model of shaken baby syndrome: preliminary characterization and evidence role of free radicals in cortical hemorrhaging and progressive neuronal degeneration. J Neurotrauma 1988; 15: 693-705.

10 Bonnier C, Mesples B, Carpentier S, Henin D, Gressens P. Delayed white matter injury in a murine model of shaken baby syndrome. Brain Pathol 2002; 12: 320-328.

11 May PR, Fuster JM, Haber J, Hirschman A. Woodpecker drilling behavior, an endorsement of the rotational theory of impact brain injury. Arch Neurol 1979; 36: 370-373

12 Sielman H. My year with the woodpeckers (translated from German by Sidney Lightman). Barrie and Rockliff: London, 1994.

13 Becher F. Untersuchungen an spechten zur trage der funktionellen anpassung an die mechanische belastrung. Z Naturforsch 1953; 2-203.

14 Gilliland M, Folberg R. Shaken babies - some have no impact injuries. J Forensic Sci 1996; 41: 114-116.

15 Budenz D, Farber MG, Mirchandani HG, Park H, Rorke LB. Ocular and optic nerve hemorrhages in abused infants with intracranial injuries. Ophthalmology 1994; 3: 559-565.

16 Elner SG, Elner VM, Arnall M, Albert DM. Ocular and associated systemic findings in suspected child abuse: a necroscopy study. Arch Ophthalmol 1990; 8: 1094-1101.

17 Gilliland M, Luckenbach M, Chenier T. Systemic and ocular findings in 169 prospectively studied child deaths: retinal 
hemorrhages usually mean child abuse. Forensic Sci In 1994; 68: 117-132.

18 Duke-Elder S, Wyber KC. The Eye. In: Elder D (ed). System of Ophthalmology, The Anatomy of the visual system. Henry Kimpton: London, 1961 pp 1, 2, 83.

19 Watson P. Diseases of the sclera and episclera. In: Duane TD (ed). Duane's Clinical Ophthalmology. Lippincott Williams \& Wilkins: Philadelphia, 1998 pp 4, 23.

20 Murphy CJ. Raptor ophthalmology. Comp Cont Educ Pract Vet 1987; 9: 256.

21 Schwab IR. Cure for a headache. Br J Ophthalmol 2002; 86: 843-845.

22 Weinberg H, Tunnessen W. Megacephaly: heeding the head. Contemp Pediatr 1969; 13: 169-175.

23 Andrews AP. Ocular manifestations of chid abuse. Penn Med 1996; 995: 71-75.

24 Betz M. Morphological analysis of retinal hemorrhages in the shaken baby syndrome. Forensic Sci Int 1996; 78: 71-80.
25 Dana M, Werner MS, Viana MA, Shapiro MJ. Spontanous and traumatic vitreous hemorrhage. Ophthalmology 1993; 100: 1377-1383.

26 May PRA, Fuster JM, Newman P, Hirschman A. Woodpeckers and head injury. Lancet 1976; 1: 454-455.

27 Gordon D. Woodpeckers, gannets, and head injury. Lancet 1976; 1: 801-802.

28 Burt WH. Adaptive modifications in woodpeckers. Univ Calif Publ Zool 1930; 32: 455-524.

29 Bock WJ. Kinetics of the avian skull. J Morphol 1964; 114: $1-42$.

30 Spring LW. Climbing and pecking adaptations in some North American woodpeckers. Condor 1965; 67: 457-488.

31 Duhaime AC, Alario AJ, Lewander WJ, Schut L, Sutton LN, Seidl TS et al. Head injury in very young children: mechanisms, injury types, and ophthalmologic findings in 100 hospitalized patients younger than 2 years of age. Pediatrics 1992; 90: 179-185. 